\title{
Wireless Localization Based on Deep Learning: State of Art and Challenges
}

\author{
Yun-Xia Ye, ${ }^{1,2}$ An-Nan Lu, ${ }^{1,2}$ Ming-Yi You $\mathbb{D D}^{1,2}$ Kai Huang $^{\mathbb{D}},{ }^{1,2}$ and Bin Jiang ${ }^{1,2}$ \\ ${ }^{1}$ Science and Technology on Communication Information Security Control Laboratory, Jiaxing, Zhejiang 314033, China \\ ${ }^{2}$ No. 36 Research Institute of CETC, Jiaxing, Zhejiang 314033, China \\ Correspondence should be addressed to Ming-Yi You; youmingyi@126.com
}

Received 15 July 2020; Revised 15 September 2020; Accepted 6 October 2020; Published 19 October 2020

Academic Editor: Jian Feng Li

Copyright (c) 2020 Yun-Xia Ye et al. This is an open access article distributed under the Creative Commons Attribution License, which permits unrestricted use, distribution, and reproduction in any medium, provided the original work is properly cited.

The problem of position estimation has always been widely discussed in the field of wireless communication. In recent years, deep learning technology is rapidly developing and attracting numerous applications. The high-dimension modeling capability of deep learning makes it possible to solve the localization problems under many nonideal scenarios which are hard to handle by classical models. Consequently, wireless localization based on deep learning has attracted extensive research during the last decade. The research and applications on wireless localization technology based on deep learning are reviewed in this paper. Typical deep learning models are summarized with emphasis on their inputs, outputs, and localization methods. Technical details helpful for enhancing localization ability are also mentioned. Finally, some problems worth further research are discussed.

\section{Introduction}

The attribute of target position is as important as its identity attribute. The target position is a core element in communications, logistics, and military field. Localization can be either self-positioning (e.g., target localization based on Global Positioning System (GPS)) or positioning interested objects (e.g., interference source positioning). We can either transmit a signal actively to locate the target or receive the signal from the target to determine its position. The target localization can be based on image data or signal data.

With the development of machine learning and artificial intelligence technology, some scholars began to pay attention to target localization by machine learning technology. In recent years, the powerful modeling capabilities of deep neural networks are attracting a large number of scholars to study effective use of deep learning techniques to solve the difficult problems in the field of target localization. The indoor localization problem is one of the most difficult problems. Due to the problems such as wall occlusion, the indoor localization accuracy based on GPS signals is not desirable, and sometimes the localization function is interrupted if the signal is very weak.
On the other hand, with the development of the Internet of Things, robots, etc., it is desirable to obtain higher localization accuracy even in indoor environment. Therefore, it becomes valuable and interesting to solve the problem of accurate target localization in indoor environment with large dynamics, multiple reflections, and occlusion by deep learning technology. In addition, some scholars used some deep learning techniques for outdoor large-scale sensor network localization [1], TDOA localization [2], and target tracking [3,4].

While deep learning technology is gradually applied in the field of wireless localization, a work systematically summarizing, classifying, and discussing related results has not yet been reported to our best knowledge. For the application of deep learning technology in the field of wireless localization, the main purpose of this paper is to propose some problems solved by deep learning technology, summarize the typical deep learning models, explore the input forms and localization methods, and pay attention to the technical details in literature which can help to improve localization performance. Besides, we raise some problems that need further study on the wireless localization problem. 


\section{Wireless Indoor Localization Technology Based on Deep Learning}

The serious multipath transmission environment in indoor wireless localization poses great challenges for localization modeling. The powerful modeling ability of deep neural networks provides a powerful tool to deal with this problem. In view of the wide application and urgent research on indoor localization (e.g., there are international conferences on indoor localization), the research studies on indoor localization are very abundant and the methods are very various. There are many elements of wireless indoor localization technology based on deep learning that can be classified. Indoor localization technology is divided into device-oriented indoor localization and device-free indoor localization according to whether the target carries equipment or not in this paper. Certainly, these methods can also be divided from network type, feature type, and localization method. In addition, some localization issues for sound source which are similar to the wireless indoor localization issues are also introduced.

\subsection{Device-Oriented Indoor Localization. Device-oriented} indoor localization means that a device carried by the target is used in positioning process. This device either receives the environmental signal as a receiver to position itself (the basic model is shown in Figure 1) or transmits a signal as a transmitter, and this signal is received by peripheral sensors to estimate the target position (the basic model is shown in Figure 2).

2.1.1. Self-Positioning. In 2014, Zhang et al. [5] proposed an indoor localization method based on the received wireless LAN WiFi signal strength using Deep Neural Network (DNN) and Hidden Markov Model (HMM), which modeled the indoor localization problem as a classification problem. Firstly, the received signal strengths of multiple hotspots are casted in a four-layer DNN to obtain a coarse estimate of the position. Then, a precise estimate of the position is obtained from the multiple coarse estimates by HMM processing.

In 2016, Félix et al. [6] proposed a fingerprinting indoor localization algorithm based on DNN, Deep Belief Network, and Gauss-Bernoulli Deep Belief Network Regression model by the characteristics of received signal strength. The simulation results show that the accuracy of DNN is the highest.

In 2016, Huang et al. [7] developed an indoor localization research based on deep neural networks with $\mathrm{WiFi}$, iBeacon signals, geomagnetic signals, and other signals and compared the accuracy of localization algorithms with single source data and fusion of multisource data.

In 2017, Xiao et al. [8] proposed two learning algorithms, namely, a deep learning architecture for regression and Support Vector Machine (SVM) for classification, to estimate the target position from the measured signal fingerprint which is the received signal strength from each transmitter, and particularly mentioned that the technique of data enhancement based on random perturbation (the order of signal strength from different transmitter is independent of position) can improve the localization accuracy by $10 \%$.

In 2017, Zhang et al. [9] proposed a new indoor fingerprinting localization system based on deep learning, combining received signal strength of $\mathrm{WiFi}$ and pervasive magnetic field to obtain richer fingerprinting, and investigated the indoor localization method based on deep neural networks in the form of classification and regression. At the same time, the effects of different types of neural networks, different mesh sizes, and different data on localization performance were described.

In 2018, Aikawa et al. [10] introduced an indoor localization method based on deep learning and WLAN signal. The method uses the received signal strengths of multiple WLAN access points as features to construct a six-layer neural network model. An experiment was conducted in an underground shopping center in Himeji City in Japan to validate the effectiveness of their approach. Experiment results show that the method is finer than the traditional least square method when the training time is sufficient.

In 2018, Lin et al. [11] proposed an indoor localization method based on a multitask learning network to handle the poor similarity of received signal strengths using $\mathrm{WiFi}$ signals in adjacent locations. The network mainly includes three parts. Firstly, a neural network is used for extracting robust regional features based on the received signal strength of adjacent locations (this paper considers 5 adjacent locations). Then, a neural network is used for learning consistent features to deal with the similarity inconsistency problem. Finally, the position is estimated based on a regression model.

In 2018, Liu et al. [12] pointed out that, although the current fingerprinting localization technology can obtain room level accuracy, the time-varying property of received signal strength caused a large position estimation error. Therefore, an autoencoder adopted to preprocess the noisy received signal strength is designed to improve localization accuracy. The method includes offline phase and online phase. In the offline phase, a deep autoencoder is trained to denoise the data. In the online phase, the fingerprint characteristics of received signal strength are obtained based on the weights after the autoencoder training, and the fingerprint characteristics are the input of three machine learning algorithms, which are random forest regression, multilayer perception classification, and multilayer perception regression. The final estimated position can be obtained by averaging over the results of three algorithms.

In 2018, De Vita and Bruneo [13] presented an indoor user localization method based on $\mathrm{WiFi}$ received signal strength and introduced the fingerprint data collection method in detail. The indoor user position is estimated by a DNN classification model using the Media Access Control (MAC) addresses as feature labels and using the corresponding received signal strengths as the feature value.

In 2018, Wu and Tseng [14] proposed a DNN-based indoor localization method using unsupervised preprocessing of channel state information (CSI) to extract richer features of CSI at different reference points. Then, the output of DNN which is the probabilities of received 


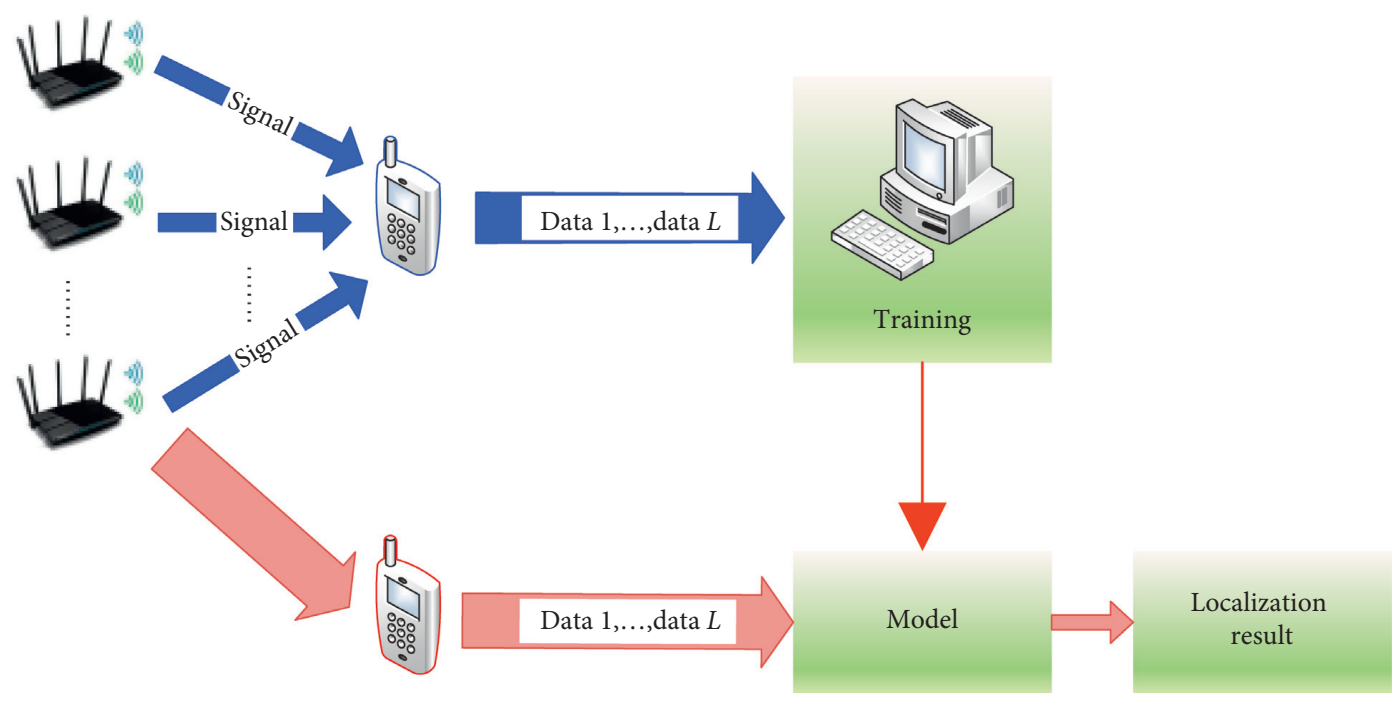

FIgURE 1: Basic model of self-positioning.

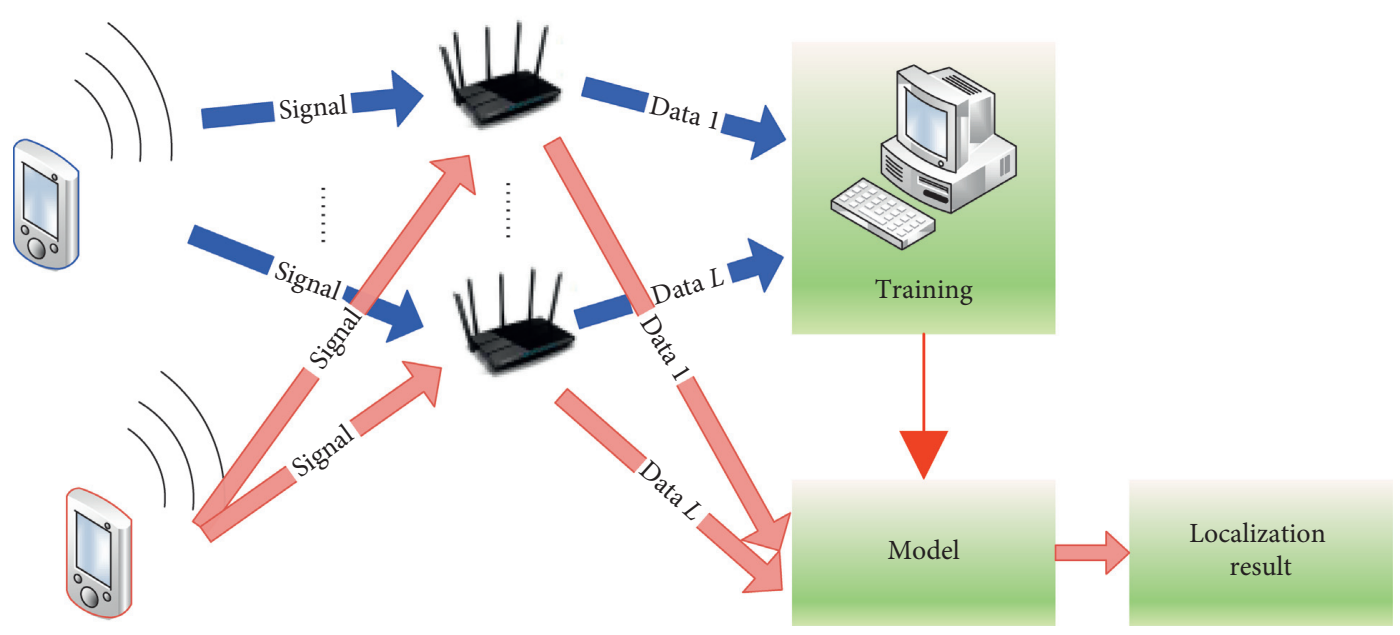

Figure 2: Basic model of passive positioning.

position at the reference points can be used for estimating the weighted average position of the receiver.

In 2018, Hsu et al. [15] proposed an adaptive indoor localization scheme based on WiFi signal strengths. The scheme can adaptively adopt the proper fingerprint database built by the crossentropy algorithm or the mean squared algorithm according to the noise level of the received signals. The Bayesian probability algorithm is used for position estimation. Experimental results show that the scheme has lower localization error whether in the noise-free environment or in the noisy environment.

In 2018, Le et al. [16] proposed a WLAN-fingerprintbased indoor localization method using unsupervised deep feature learning for the problem of small training samples. Firstly, a large number of unlabeled received signal strengths are used for extracting the deep features by the Deep Belief Network training. Then, a small number of labeled samples are used for training the shallow supervised learning algorithms (such as SVM). The experimental results show that the localization method based on a large number of unlabeled fingerprints and a small number of labeled fingerprints with deep feature learning can obtain the same localization accuracy as counterpart based on the same number of entirely labeled fingerprints with shallow feature learning.

In 2018, Khatab et al. [17] also studied on the indoor localization in wireless sensor networks based on the received signal strength. Aiming at the problem that the deep neural networks are training slowly, a deep extreme learning machine is proposed for training. To deal with the dynamic environment which leads to the time-varying characteristics of the received signal strength, they increased the number of training data and used high-level features by autoencoder to train the model.

In 2018, Dou et al. [18] pointed out that WiFi fingerprints localization technology based on the received signal strength is widely used in vast indoor localization systems due to the low cost and high localization accuracy. However, the fluctuation of wireless signal will cause a great fluctuation 
on received signal strength, which poses great challenges to indoor localization based on WiFi fingerprints. Dou et al. proposed a top-down searching method based on deep reinforcement learning to deal with the dynamic environment in WiFi fingerprints indoor localization. The localization method based on deep reinforcement learning has three advantages. Firstly, the method can be readily applied to the fluctuation in received signal strength due to dynamic environment. Secondly, the learning process can be online to realize real-time positioning. Thirdly, it is not necessary to require the prior knowledge of floor plan.

In 2019, Elbes et al. [19] presented an indoor localization approach based on WiFi received signal strengths and Long Short-Term Memory (LSTM) Neural Networks. The experimental results show that the localization approach is effective in corridors of the L-shaped floor.

In 2019, Ebuchi and Yamamoto [20] described a smart parking system based on a small number of beacon devices. The system uses the beacon signal strength measured by the smartphones to locate vehicles and pedestrians, and then the gateway broadcasts the position information to all smartphones in the parking lot so that contact accidents can be avoided. A full connected layer DNN and Convolutional Neural Network classification model is used for locating vehicles and pedestrians. The experimental results show that estimation accuracy of the vehicle's position is higher than $98 \%$, and estimation accuracy of the pedestrian's position is about $70 \%$.

In 2019, Jiang et al. [21] proposed fingerprinting-based radio-frequency identification (RFID) indoor localization algorithm based on deep belief network. The collected received signal strength data is input into a deep belief network to extract deep features and then the target is located by the similarity comparison with the deep features.

In 2019, Bae and Choi [22] proposed an indoor localization method based on LSTM Neural Network using continuous geomagnetic data. The localization accuracy of LSTM Neural Network is higher than that of classical recurrent neural network and the existing RF-based fingerprinting techniques.

In 2019, Rizk et al. [23] pointed out that WiFi-based indoor localization technology relies on WiFi chips which limit wide application of the technology. In addition, technologies based on inertial sensors such as accelerometers, gyroscopes, and compasses are only available on highend phones. Therefore, they proposed an indoor localization method based on cellular signals received by mobile phones. And they proposed two training data augmentation techniques: random augmenter and lower-bound cropper.

In 2019, Abbas et al. [24] pointed out that many indoor localization systems have been proposed relying on $\mathrm{WiFi}$ fingerprinting due to the wide use of indoor WiFi. However, due to the inherent noise and instability of wireless signals, the localization accuracy usually degrades. So, they proposed a deep learning-based indoor localization system called WiDeep to obtain high accuracy and robustness. An autoencoder is trained for the signal strength of each access point separately, and the position estimation is obtained by a probabilistic fusion algorithm based on the output of latent features from these autoencoders. It is worth noting that Abbas et al. [24] also introduce two techniques adding stochastic noise to the input training data in order to improve the robustness of WiDeep in noisy environments.

In 2017, Takeda and Komatani [25] investigated a sound source localization method based on deep neural networks when sound source is at an unknown position in unknown reverberant environments. The sound source localization accuracy can be improved by using unsupervised adaption of parameters and early stopping of the parameter update.

In 2019, He et al. [26] pointed out that the DNN became the main approaches for sound source localization and directions of arrival estimation, but these approaches still have two major drawbacks: (1) these approaches require a large amount of training data for specific devices and it is particularly difficult to obtain the true sound source positions, and (2) these methods are very sensitive to the mismatch between the training and test conditions. To address the problems, they have studied two domain adaptation methods and their combination for multiple sound source localization: weak supervision and domain adversarial training. The weak supervision regularizes the output of neural network and makes it closer to the possible output space based on the inexact labels. The domain adversarial training aims to find domain-invariant features. The experimental results show that the neural network model adapted with the weak supervision has been significantly improved, but the domain adversarial training does not further improve the performance of the model.

In 2019, Xu et al. [27] proposed a biologically inspired binaural sound localization system for reverberant environments. This sound source localization system uses a convolutional neural network to analyze the 2-D correlation matrix generated by the stereo cochlear system. The system can obtain a lower average absolute error in the $-90^{\circ}$ to $90^{\circ}$ range.

2.1.2. Passive Positioning. In 2017, Xue et al. [28] proposed a localization algorithm based on deep learning and asynchronous time difference of arrival (TDOA) data, measurement errors, and missing data. The algorithm determines the target state first and then predicts the asynchronous TDOAs with measurement errors or missing data. Finally, the target is located based on the predicted TDOA data.

In 2018, Berruet et al. [29] proposed an indoor localization method based on CSI and convolutional neural network in Internet of Things context. This method uses different subcarriers and different receiving antenna CSI to form a two-dimensional matrix as the input of the neural network and uses the target position as the output (regression model). In this method, the receiver is a gateway with multiple antenna elements, while the transmitter is a device with one antenna element, so only one receiver is needed to estimate the target position.

In 2019, Chen et al. [30] used the WiFi routers to scan smartphones so that a large amount of received signal strengths can be collected. Then, they extracted the local 
features by consecutive received signal strength features for each router. The LSTM network is leveraged to learn highlevel representations from the extracted local features and indoor localization was realized by a regression model.

2.2. Device-free Indoor Localization. Device-free indoor localization means that the located target does not carry any receiving or transmitting equipment (basic model is shown as Figure 3). The localization system achieves the location estimate by utilizing the target's influence on the environment (e.g., communication channel).

In 2016, Wang et al. [31] proposed PhaseFi, an indoor localization system based on calibrated CSI phase information. In PhaseFi, firstly, the raw phase information is extracted from the multiple antennas and multiple subcarriers of the IEEE802.11n network interface card. Then, a linear transformation is applied to extract the calibrated phase information. In the offline phase, a three-layer neural network is designed to train the calibrated phase information, and the weights of the neural network are used as fingerprint features. In the online phase, a probabilistic method based on radial basis functions is used for estimating the target position.

Wang et al. [32,33] studied an indoor localization scheme based on CSI information instead of CSI phase information [28].

In 2017, Wang et al. [34] proposed an indoor localization method ( $\mathrm{CiFi}$ ) based on deep convolutional neural networks. The method uses one transmitter and some receivers consisting of three direction-finding antennas. The AOA (angle of arriving) values of different subcarriers of WiFi signal are used for forming CSI AOA images, and the images are uses as the input of the neural network.

In 2017, in order to improve the localization accuracy, Wang et al. [35] used bimodal CSI including AOA and CSI amplitude to estimate indoor position. At the same time, in order to reduce the training time and data storage requirement, a deep residual sharing learning network was used for model training. The input of the network includes two channels, and each input tensor of each signal includes three images: two of them are AOA-time images obtained from three antennas and the other is a CSI amplitude-time image from one antenna. Experimental results show that the localization accuracy of this method is significantly improved.

In 2019, Abdul Samadh et al. [36] pointed out that the advantages of device-free indoor localization methods include the following: (1) they can be used for tracking targets that cannot be controlled directly, (2) they can be used for locating in unexpected circumstances, and (3) they are comparatively cost-effective with minimal infrastructure setup requirements. They studied the localization method based on CSI amplitude and convolutional neural network (classification model) and also studied the impact of CSI time-varying characteristics on localization accuracy. The results show that CSI time-varying characteristics have a greater influence on localization accuracy using CSI amplitude information only.
In 2019, Hsieh et al. [37] formulated the indoor localization as a classification problem, and developed four indoor localization methods based on multilayer perceptron, one-dimensional convolutional neural network, received signal strength, and CSI. The localization method based on one-dimensional convolutional neural network is proposed for the first time. The experimental results show that the onedimensional convolutional network using CSI information achieves excellent localization performance with much lower network complexity.

In 2019, Liu et al. [38] pointed out that deep neural networks have shown great potential in indoor high-precision localization, but the inner principles are not adequately understood. So, they provide quantitative and visual explanations for the deep learning process and the important features learnt by deep neural network during the learning process. Several visualization techniques are proposed including dimensionality reduction visualization, visual analysis, and information visualization.

\section{Wireless Outdoor Localization Technology Based on Deep Learning}

Compared with the indoor localization problem, there are even more issues associated with outdoor localization. The multipath effect is greatly reduced in outdoor localization, so the contradiction of localization modeling is not as serious as that of indoor localization. Therefore, while paying attention to the methods, we should also pay attention to the motivation and purpose of the research. Basic models of outdoor localization are the same as those for indoor localization, as shown in Figures 1-3.

In 2017, Houégnigan et al. [39] pointed out that, although underwater range can be standardly estimated by widely spaced sensors in the higher frequency ranges and assuming direct path, opportunistically estimated using surface and bottom reflection or using modal decomposition at certain low frequencies, and it remains a big challenge to develop a general system based on a single sensor or a smallaperture array that can adapted to real time. They introduced the early results of their ongoing underwater localization and sound source range estimation based on a single sensor and the experimental results of range estimation using shallow and deep neural networks by a single sensor. The deep neural networks used in [39] are AlexNet, VGG-16, and VGG-19.

In 2019, Chang [40] proposed a threshold adaptive varied method for tracking based on deep learning to improve Extended Kalman Filter (EKF) algorithm because the tracking threshold is difficult to update adaptively which may lead to a divergence when tracking a high-speed target. This method uses a recurrent neural network model. The model inputs include current and previous position estimation, and the model output is the target region. If the target region estimation of improved EKF is inconsistent with the neural network model, the tracking threshold should be adjusted until they are consistent.

Existing telecommunication-based localization methods suffer from the challenges of either high localization errors or requiring intensive data samples or high sensitivity 


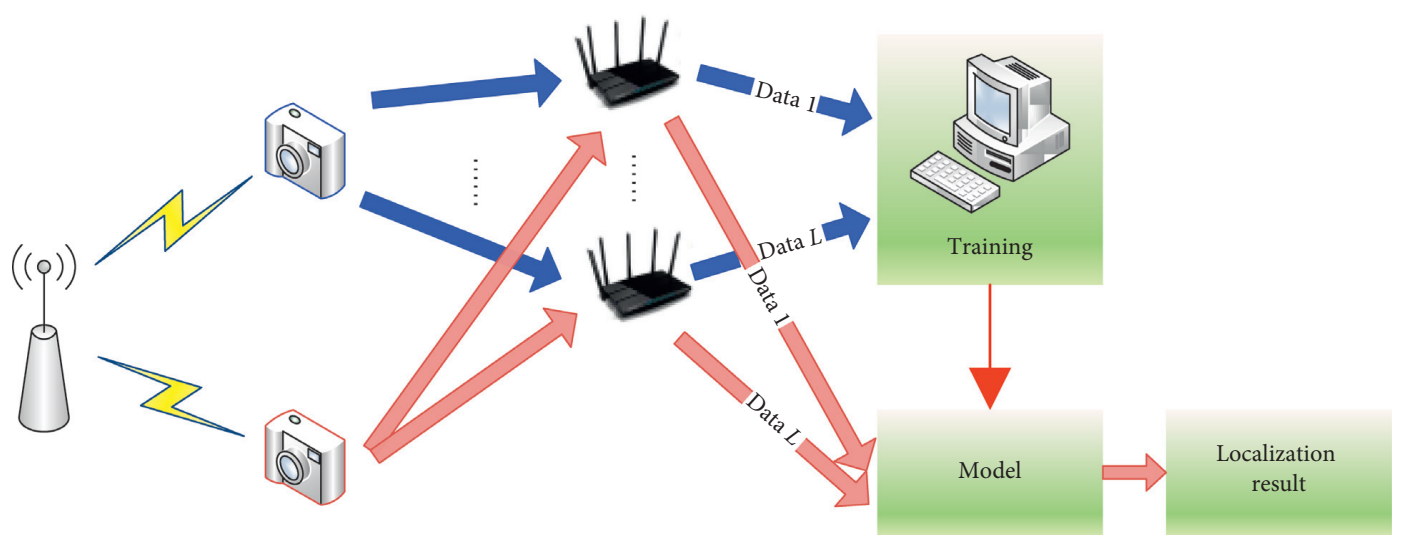

FIgURE 3: Basic model of device-free indoor localization.

towards noisy Measurement Record (MR) data. To address the challenges, Zhang [41] proposed a telecommunication localization framework. The framework mainly includes three main parts: a localization model, a method overcoming the data scarcity issue, and a method repairing noisy MR samples. The localization models include single-point-based localization model and sequence-based localization model. The idea of transfer learning is adopted to overcome the data scarcity issue. In addition, a confidence level method is proposed to detect those MR samples with high localization errors by confidence levels and then repair the predicted positions.

In 2019, Shen et al. [42] proposed a relative localization method of high-density RFID tags via phase and received signal strength indication (RSSI) based on deep learning with convolutional neural networks.

\section{Discussion and Conclusion}

At present, the core elements of indoor localization technology based on deep learning include the following. (1) Application signal types: WiFi, WLAN, iBeacon, geomagnetic signals, base station cellular signals, etc. (2) Feature types: received signal strength, CSI (amplitude and phase), angle of arrival etc. (3) Preprocessing methods: normalization, adding noise, training data increase, high-level feature learning based on autoencoder, etc. (4) Network frameworks: convolutional neural networks, LSTM neural networks, fully connected networks, etc. (5) Localization methods: classification methods based on neural networks, regression methods based on neural networks, probability methods based on higher-level features, etc. Although there are research results, it is difficult to evaluate the localization performance of various methods based on a unified standard at present. Therefore, further research on the optimal combination of these will be a valuable research direction.

The major challenges of indoor localization are the environmental interference and the time-varying characteristics of the channel, so the transfer function between position and position-sensitive parameters cannot be established. However, the nonideal model can be solved by deep learning because of the high-dimension modeling capabilities. At present, a mount of methods based on increasing the training samples, extracting higher-level features, and using deep reinforcement learning have been proposed. It is necessary to compare the cost and performance of the methods with unified data and to find better approaches.

The complex environment in indoor localization greatly promotes the application and research on deep learning technology in indoor localization. In outdoor localization, the first thing that deep learning needs to solve is the application requirement. Although a few researches have been carried out, it is difficult to conclude whether the application of deep learning technology is the best choice in these problems.

At present, most research studies on deep learning-based localization problems focus on two-dimensional problems. For three-dimensional localization problems, the cost of collecting training samples will greatly increase. Therefore, it is of great significance to research on how to reduce the demand of training samples for three-dimensional localization.

Compared with the indoor wireless environment, the complexity of the underwater environment is increased. Making full use of the technical achievements on indoor localization, deep learning technology may be a powerful tool to deal with underwater precise localization.

This article focuses on the application of deep learning technology in the field of wireless localization, the problems solved by deep learning technology, the common deep learning models, the input form, and the methods. And we discuss the need for further research on wireless localization. With the advance and development of big data and deep learning technology, better performance and results are expected to be obtained from wireless localization systems in complex practical application environments.

\section{Conflicts of Interest}

The authors declare that they have no conflicts of interest. 


\section{Acknowledgments}

This work was support by No. 36 Research Institute of CETC under the project no. CX05.

\section{References}

[1] B. G. Maciel-Pearson, S. Akçay, A. Atapour-Abarghouei, C. Holder, and T. P. Breckon, "Multi-task regression-based learning for autonomous unmanned aerial vehicle flight control within unstructured outdoor environments," IEEE Robotics and Automation Letters, vol. 4, no. 4, pp. 4116-4123, 2019.

[2] F. Carrino, A. Janka, O. Abou Khaled, and E. Mugellini, "LoRaLoc: machine learning-based fingerprinting for outdoor geolocation using LoRa," in Proceedings of the 2019 6th Swiss Conference on Data Science (SDS), pp. 82-86, Bern, Switzerland, June 2019.

[3] G. Yang and Z. Chen, "Pedestrian tracking algorithm for dense crowd based on deep learning," in Proceedings of the 2019 6th International Conference on Systems and Informatics (ICSAI), pp. 568-572, Shanghai, China, November 2019.

[4] L. Li and S. Qin, "A synthetic integrative algorithm of fast concurrent detection-recognition with effective tracking of aerial objects based on deep learning of CNN," in Proceedings of the IEEE 4th Advanced Information Technology, Electronic and Automation Control Conference (IAEAC), pp. 1187-1194, Chengdu, China, December 2019.

[5] W. Zhang, K. Liu, W. Zhang, Y. Zhang, and J. Gu, "Wi-Fi positioning based on deep learning," in Proceedings of the 2014 IEEE International Conference on Information and Automation (ICIA), pp. 1176-1179, Hailar, China, July 2014.

[6] G. Félix, M. Siller, and E. N. Álvarez, "A fingerprinting indoor localization algorithm based deep learning," in Proceedings of the 2016 Eighth International Conference on Ubiquitous and Future Networks (ICUFN), pp. 1006-1011, Vienna, Austria, July 2016.

[7] L. Huang, X. Gan, S. Li, H. Zhang, Y. Li, and R. Zhu, "Indoor positioning technology based on deep neural networks," in Proceedings of the 2018 Ubiquitous Positioning, Indoor Navigation and Location-Based Services (UPINLBS), pp. 1-6, Wuhan, China, March 2018.

[8] L. Xiao, A. Behboodi, and R. Mathar, "A deep learning approach to fingerprinting indoor localization solutions," in Proceedings of the 2017 27th International Telecommunication Networks and Applications Conference (ITNAC), pp. 1-7, Melbourne, Australia, November 2017.

[9] W. Zhang, R. Sengupta, J. Fodero, and X. Li, "DeepPositioning: intelligent fusion of pervasive magnetic field and $\mathrm{Wi}$-Fi fingerprinting for smartphone indoor localization via deep learning," in Proceedings of the 2017 16th IEEE International Conference on Machine Learning and Applications, pp. 7-13, Cancun, Mexico, December 2017.

[10] S. Aikawa, S. Yamamoto, and M. Morimoto, "WLAN finger print localization using deep learning," in Proceedings of the 2018 IEEE Asia-Pacific Conference on Antennas and Propagation (APCAP), pp. 541-542, Auckland, New Zealand, August 2018.

[11] W. Lin, C. Huang, N. Duc, and H. Manh, "Wi-Fi indoor localization based on multi-task deep learning," in Proceedings of the 2018 IEEE 23rd International Conference on Digital Signal Processing (DSP), pp. 1-5, Shanghai, China, November 2018.
[12] J. Liu, N. Liu, Z. Pan, and X. You, "AutLoc: deep autoencoder for indoor localization with RSS fingerprinting," in Proceedings of the 2018 10th International Conference on Wireless Communications and Signal Processing (WCSP), pp. 1-6, Hangzhou, China, October 2018.

[13] F. De Vita and D. Bruneo, "A deep learning approach for indoor user localization in smart environments," in Proceedings of the 2018 IEEE International Conference on Smart Computing (SMARTCOMP), pp. 89-96, Taormina, Italy, June 2018.

[14] G. Wu and P. Tseng, "A deep neural network-based indoor positioning method using channel state information," in Proceedings of the 2018 International Conference on Computing, Networking and Communications (ICNC), pp. 290294, Maui, HI, USA, March 2018.

[15] C. Hsu, Y. Chen, T. Juang, and Y. Wu, "An adaptive Wi-fi indoor localization scheme using deep learning," in Proceedings of the 2018 IEEE Asia-Pacific Conference on Antennas and Propagation (APCAP), pp. 132-133, Auckland, New Zealand, August 2018.

[16] D. V. Le, N. Meratnia, and P. J. M. Havinga, "Unsupervised deep feature learning to reduce the collection of fingerprints for indoor localization using deep belief networks," in Proceedings of the 2018 International Conference on Indoor Positioning and Indoor Navigation (IPIN), pp. 1-7, Nantes, France, September 2018.

[17] Z. E. Khatab, A. Hajihoseini, and S. A. Ghorashi, "A fingerprint method for indoor localization using autoencoder based deep extreme learning machine," IEEE Sensors Letters, vol. 2, no. 1, pp. 1-4, 2018.

[18] F. Dou, J. Lu, Z. Wang, X. Xiao, J. Bi, and C. Huang, "Topdown indoor localization with Wi-fi fingerprints using deep Q-network," in Proceedings of the 2018 IEEE 15th International Conference on Mobile Ad Hoc and Sensor Systems (MASS), pp. 166-174, Chengdu, China, October 2018.

[19] M. Elbes, E. Almaita, T. Alrawashdeh, T. Kanan, S. AlZu'bi, and B. Hawashin, "An indoor localization approach based on deep learning for indoor location-based services," in Proceedings of the 2019 IEEE Jordan International Joint Conference on Electrical Engineering and Information Technology (JEEIT), pp. 437-441, Amman, Jordan, April 2019.

[20] T. Ebuchi and H. Yamamoto, "Vehicle/pedestrian localization system using multiple radio beacons and machine learning for smart parking," in Proceedings of the 2019 International Conference on Artificial Intelligence in Information and Communication (ICAIIC), pp. 86-91, Okinawa, Japan, February 2019.

[21] H. Jiang, C. Peng, and J. Sun, "Deep belief network for fingerprinting-based RFID indoor localization," in Proceedings of the ICC 2019-2019 IEEE International Conference on Communications (ICC), pp. 1-5, Shanghai, China, May 2019.

[22] H. J. Bae and L. Choi, "Large-scale indoor positioning using geomagnetic field with deep neural networks," in Proceedings of the ICC 2019-2019 IEEE International Conference on Communications (ICC), pp. 1-6, Shanghai, China, May 2019.

[23] H. Rizk, M. Torki, and M. Youssef, "CellinDeep: robust and accurate cellular-based indoor localization via deep learning," IEEE Sensors Journal, vol. 19, no. 6, pp. 2305-2312, 2019.

[24] M. Abbas, M. Elhamshary, H. Rizk, M. Torki, and M. Youssef, "WiDeep: WiFi-based accurate and robust indoor localization system using deep learning," in Proceedings of the 2019 IEEE International Conference on Pervasive Computing and Communications (PerCom), pp. 1-10, Kyoto, Japan, March 2019. 
[25] R. Takeda and K. Komatani, "Unsupervised adaptation of deep neural networks for sound source localization using entropy minimization," in Proceedings of the 2017 IEEE International Conference on Acoustics, Speech and Signal Processing (ICASSP), pp. 2217-2221, New Orleans, LA, USA, March 2017.

[26] W. He, P. Motlicek, and J. Odobez, "Adaptation of multiple sound source localization neural networks with weak supervision and domain-adversarial training," in Proceedings of the ICASSP 2019-2019 IEEE International Conference on Acoustics, Speech and Signal Processing (ICASSP), pp. 770774, Brighton, UK, May 2019.

[27] Y. Xu, S. Afshar, R. K. Singh, T. J. Hamilton, and A. van Schaik, "A machine hearing system for binaural sound localization based on instantaneous correlation," in Proceedings of the 2018 IEEE International Symposium on Circuits and Systems (ISCAS), Florence, Italy, May 2018.

[28] Y. Xue, W. Su, H. Wang, D. Yang, and Y. Jiang, "DeepTAL: deep learning for TDOA-based asynchronous localization security with measurement error and missing data," IEEE Access, vol. 7, pp. 122492-122502, 2019.

[29] B. Berruet, O. Baala, A. Caminada, and V. Guillet, "DelFin: a deep learning based CSI fingerprinting indoor localization in IoT context," in Proceedings of the 2018 International Conference on Indoor Positioning and Indoor Navigation (IPIN), pp. 1-8, Nantes, France, September 2018.

[30] Z. Chen, H. Zou, J. Yang, H. Jiang, and L. Xie, "Wi-Fi fingerprinting indoor localization using local feature-based deep LSTM," IEEE Systems Journal, vol. 14, no. 2, pp. 3001-3010, 2019.

[31] X. Wang, L. Gao, and S. Mao, "CSI phase fingerprinting for indoor localization with a deep learning approach," IEEE Internet of Things Journal, vol. 3, no. 6, pp. 1113-1123, 2016.

[32] X. Wang, L. Gao, S. Mao, and S. Pandey, "DeepFi: deep learning for indoor fingerprinting using channel state information," in Proceedings of the 2015 IEEE Wireless Communications and Networking Conference (WCNC), pp. 1666-1671, New Orleans, LA, USA, March 2015.

[33] X. Wang, L. Gao, S. Mao, and S. Pandey, "CSI-based fingerprinting for indoor localization: a deep learning approach," IEEE Transactions on Vehicular Technology, vol. 66, no. 1, pp. 763-776, 2016.

[34] X. Wang, X. Wang, and S. Mao, "CiFi: deep convolutional neural networks for indoor localization with $5 \mathrm{GHz} \mathrm{Wi}-\mathrm{Fi}$," in Proceedings of the 2017 IEEE International Conference on Communications (ICC), pp. 1-6, Paris, France, May 2017.

[35] X. Wang, X. Wang, and S. Mao, "ResLoc: deep residual sharing learning for indoor localization with CSI tensors," in Proceedings of the 2017 IEEE 28th Annual International Symposium on Personal, Indoor, and Mobile Radio Communications (PIMRC), pp. 1-6, Montreal, Canada, October 2017.

[36] S. Abdul Samadh, Q. Liu, X. Liu, N. Ghourchian, and M. Allegue, "Indoor localization based on channel state information," in Proceedings of the 2019 IEEE Topical Conference on Wireless Sensors and Sensor Networks (WiSNet), pp. 1-4, Orlando, FL, USA, January 2019.

[37] C. Hsieh, J. Chen, and B. Nien, "Deep learning-based indoor localization using received signal strength and channel state information," IEEE Access, vol. 7, pp. 33256-33267, 2019.

[38] S. Liu, R. Y. Chang, and F. Chien, "Analysis and visualization of deep neural networks in device-free Wi-Fi indoor localization," IEEE Access, vol. 7, pp. 69379-69392, 2019.

[39] L. Houégnigan, P. Safari, C. Nadeu, M. André, and M. van der Schaar, "Machine and deep learning approaches to localization and range estimation of underwater acoustic sources," in Proceedings of the 2017 IEEE/OES Acoustics in Underwater Geosciences Symposium (RIO Acoustics), pp. 1-6, Rio de Janeiro, Brazil, July 2017.

[40] S. Chang, "A deep learning approach for localization systems of high-speed objects," IEEE Access, vol. 7, pp. 96521-96530, 2019.

[41] Y. Zhang, "Outdoor localization framework with telco data," in Proceedings of the 2019 20th IEEE International Conference on Mobile Data Management (MDM), pp. 395-396, Hong Kong, China, June 2019.

[42] L. Shen, Q. Zhang, J. Pang, H. Xu, and P. Li, "PRDL: relative localization method of RFID tags via phase and RSSI based on deep learning," IEEE Access, vol. 7, pp. 20249-20261, 2019. 